\title{
Cosmic Divergence, Weak Cosmic Conver- gence, and Fixed Points at Infinity
}

\author{
Ernest K. Ryu
}

\begin{abstract}
To characterize the asymptotic behavior of fixed-point iterations of non-expansive operators with no fixed points, Bauschke et al. [J. Fixed Point Theory Appl. 18:2 (2016), 297-307] recently studied cosmic convergence and conjectured that cosmic convergence always holds. This paper presents a cosmically divergent counter example, which disproves this conjecture. This paper also demonstrates, with a counter example, that cosmic convergence can be weak in infinite dimensions. Finally, this paper shows positive results relating to cosmic convergence that provide an interpretation of cosmic accumulation points as fixed points at infinity.
\end{abstract}

Mathematics Subject Classification (2010). Primary 47H09; Secondary $90 \mathrm{C} 25$.

Keywords. Cosmic convergence, non-expansive mapping, Convex optimization, Weak convergence, Minimal displacement vector.

\section{Introduction}

Let $\mathcal{H}$ be a (finite or infinite dimensional) Hilbert space with an inner product $\langle\cdot, \cdot\rangle$ and induced norm $\|\cdot\|$. Let $T: \mathcal{H} \rightarrow \mathcal{H}$ be a non-expansive operator with no fixed point. Consider the "fixed-point" iteration

$$
x^{k+1}=T\left(x^{k}\right)
$$

for some starting point $x^{0} \in \mathcal{H}$, even though $T$ has no fixed point. Since $T$ has no fixed point, $\left\{x^{k}\right\}_{k \geq 0}$ diverges in that $\left\|x^{k}\right\| \rightarrow \infty$ [10, Corollary 6(c)].

Define

$$
\operatorname{ran}(I-T)=\{x-T(x) \mid x \in \mathcal{H}\},
$$

and write $\overline{\operatorname{ran}(I-T)}$ for the closure of $\operatorname{ran}(I-T)$. Define the minimal displacement vector of $T$ as




which exists and is unique [10, Lemma 4]. Pazy, in 1971 [10, Theorem 2], elegantly characterized the asymptotic behavior of iteration (1.1) by showing

$$
x^{k}=-k v+o(k)
$$

as $k \rightarrow \infty$. Furthermore, Baillon et al., in 1978 [2, Corollary 2.3], showed

$$
x^{k+1}-x^{k} \rightarrow-v
$$

as $k \rightarrow \infty$. When $v \neq 0$, we have $\left\|x^{k}\right\|=O(k)$, and these results provide a very concrete understanding of the manner in which $x^{k}$ diverges to infinity.

When $v=0$, Pazy's result implies $x^{k}=o(k)$ without any characterization of direction and $\left\|x^{k}\right\| \rightarrow \infty$ at the slower rate of $\left\|x^{k}\right\|=o(k)$ as $k \rightarrow \infty$. This is less concrete and less satisfactory. While there has been some work since Pazy studying this case [ 8, 6, 4, our understanding is still incomplete.

In this paper, we focus on the case $v=0$; unless stated otherwise, assume $T: \mathcal{H} \rightarrow \mathcal{H}$ is non-expansive and $0 \in \overline{\operatorname{ran}(I-T)} \backslash \operatorname{ran}(I-T)$.

We say $q$ is the weak cosmic limit of $\left\{x^{k}\right\}_{k \geq 0} \subset \mathcal{H}$ if $\left\|x^{k}\right\| \rightarrow \infty$ and

$$
\left(1 /\left\|x^{k}\right\|\right) x^{k} \rightarrow q,
$$

where $\rightarrow$ denotes weak convergence. We call the weak accumulation points of $\left(1 /\left\|x^{k}\right\|\right) x^{k}$ the weak cosmic accumulation points. The strong cosmic limit and strong cosmic accumulation points are defined similarly.

As a way to understand the asymptotic behavior of the $\left\{x^{k}\right\}_{k \geq 0}$ sequence of (1.1), Bauschke et al. 4] studied the cosmic accumulation points in finite dimensional Hilbert spaces and postulated the cosmic convergence conjecture, which states $\left\{x^{k}\right\}_{k \geq 0}$ always cosmically converges.

\subsection{Contribution and organization of this paper}

This paper furthers the study of cosmic convergence and cosmic accumulation points and thereby furthers the understanding of the asymptotic behavior of the $\left\{x^{k}\right\}_{k \geq 0}$ sequence of (1.1) for non-expansive operators $T$ such that $0 \in \overline{\operatorname{ran}(I-T)} \backslash \operatorname{ran}(I-T)$.

Section 2 sets up basic preliminary information.

Section 3 presents a counter example to the cosmic convergence conjecture. There is a (firmly) non-expansive operator $T: \mathbb{R}^{2} \rightarrow \mathbb{R}^{2}$ such that its fixed-point iteration sequence does not have a cosmic limit. This shows that the cosmic convergence conjecture is false without any further assumptions.

Section 4 presents a non-expansive operator on an infinite dimensional Hilbert space for which its fixed-point iteration sequence cosmically converges to 0 weakly. This example illustrates the additional complexity of analyzing cosmic convergence in infinite dimensions.

Section 5 presents positive results towards characterizing cosmic accumulation points. In particular, we show that a non-zero cosmic limit defines a separating hyperplane between $\overline{\operatorname{ran}(I-T)}$ and $\{0\}$ and can be interpreted as a fixed point at infinity. Section 5.3 relates the presented results to the works of Lins [8] Gaubert and Vigeral [6], and Bauschke et al. [4]. 


\section{Preliminaries}

Write $\rightarrow$ and $\rightarrow$ for weak and strong convergence, respectively. Given a sequence $\left\{q^{k}\right\}_{k \geq 0} \subset \mathcal{H}$, we say $q$ is a weak accumulation point if there is a subsequence $\left\{q^{k_{j}}\right\}_{j \geq 0}$ such that $q^{k_{j}} \rightarrow q$. A strong accumulation point is defined similarly.

Lemma 2.1. Assume two sequences $\left\{x^{k}\right\}_{k \geq 0} \subset \mathcal{H}$ and $\left\{y^{k}\right\}_{k \geq 0} \subset \mathcal{H}$ satisfy

$$
\left\|x^{k}\right\| \rightarrow \infty, \quad\left\|y^{k}\right\| \rightarrow \infty
$$

as $k \rightarrow \infty$ and

$$
\left\|x^{k}-y^{k}\right\| \leq C
$$

for all $k=0,1,2, \ldots$ for some $C \geq 0$. Then $\left\{\left(1 /\left\|x^{k}\right\|\right) x^{k}\right\}_{k \geq 0}$ and $\left\{\left(1 /\left\|y^{k}\right\|\right) y^{k}\right\}_{k \geq 0}$ have the same (weak or strong) accumulation points.

Proof. The reverse triangle inequality tells us

$$
\left|\left\|x^{k}\right\|-\left\|y^{k}\right\|\right| \leq\left\|x^{k}-y^{k}\right\| \leq C .
$$

Dividing by $\left\|y^{k}\right\|$ (which is non-zero for large $k$ ) we get

$$
\left|\left\|x^{k}\right\| /\left\|y^{k}\right\|-1\right| \leq C /\left\|y^{k}\right\| \text {. }
$$

Since $\left\|y^{k}\right\| \rightarrow \infty$ as $k \rightarrow \infty$, we conclude that $\left\|x^{k}\right\| /\left\|y^{k}\right\| \rightarrow 1$.

Now we have

$$
\begin{aligned}
& \left\|\left(1 /\left\|x^{k}\right\|\right) x^{k}-\left(1 /\left\|y^{k}\right\|\right) y^{k}\right\|^{2} \\
& \quad=\left(1 /\left\|x^{k}\right\|^{2}\right)\left\|x^{k}-y^{k}+y^{k}-\left(\left\|x^{k}\right\| /\left\|y^{k}\right\|\right) y^{k}\right\|^{2} \\
& \quad \leq\left(2 /\left\|x^{k}\right\|^{2}\right)\left\|x^{k}-y^{k}\right\|^{2}+\left(2 /\left\|x^{k}\right\|^{2}\right)\left\|y^{k}-\left(\left\|x^{k}\right\| /\left\|y^{k}\right\|\right) y^{k}\right\|^{2} \\
& \quad \leq\left(2 /\left\|x^{k}\right\|^{2}\right) C^{2}+\left(2\left\|y^{k}\right\|^{2} /\left\|x^{k}\right\|^{2}\right)\left(1-\left\|x^{k}\right\| /\left\|y^{k}\right\|\right)^{2} \\
& \quad \rightarrow 0 .
\end{aligned}
$$

Thus we conclude $\left\{\left(1 /\left\|x^{k}\right\|\right) x^{k}\right\}_{k \geq 0}$ and $\left\{\left(1 /\left\|y^{k}\right\|\right) y^{k}\right\}_{k \geq 0}$ have the same accumulation points.

Lemma 2.2. Consider the fixed-point iteration (1.1) and its sequence $\left\{x^{k}\right\}_{k \geq 0}$. The set of weak accumulation points of $\left\{\left(1 /\left\|x^{k}\right\|\right) x^{k}\right\}_{k \geq 0}$ is the same irrespective of the starting point $x^{0} \in \mathcal{H}$.

Proof. In addition to $\left\{x^{k}\right\}_{k \geq 0}$, consider another sequence

$$
y^{k+1}=T\left(y^{k}\right)
$$

with some starting point $y^{0} \in \mathcal{H}$. As $T$ has no fixed point, $\left\|x^{k}\right\| \rightarrow \infty$ and $\left\|y^{k}\right\| \rightarrow \infty$ [10, Corollary 6(c)]. By non-expansiveness of $T$, we have

$$
\left\|x^{k}-y^{k}\right\| \leq\left\|x^{0}-y^{0}\right\| \text {. }
$$

The statement now follows from Lemma 2.1. 
With Lemma 2.2 we can define

$$
Q=\text { weak accumulation points of }\left(1 /\left\|x^{k}\right\|\right) x^{k},
$$

where $\left\{x^{k}\right\}_{k \geq 0}$ is the sequence of 1.1 . So $Q$ is the set of cosmic accumulation points of $T$, and we have cosmic convergence when $Q$ is a singleton.

Note that $Q$ may not necessarily be a subset of the unit sphere. Write $S$ and $B$ for the unit sphere and closed unit ball of $\mathcal{H}$, i.e.,

$$
S=\{x \in \mathcal{H} \mid\|x\|=1\}, \quad B=\{x \in \mathcal{H} \mid\|x\| \leq 1\} .
$$

Fact 2.3. If $\mathcal{H}$ is finite dimensional, $S$ and $B$ are compact. If $\mathcal{H}$ is infinite dimensional, $B$ is weakly compact but $S$ is not, and $S$ is weakly dense in $B$.

The first assertion of Fact 2.3 follows from the Heine-Borel theorem. The second assertion of Fact 2.3 can be found in standard references such as [1. Corollary 6.29].

When $\mathcal{H}$ is finite dimensional $Q \subseteq S$. When $\mathcal{H}$ is infinite dimensional $Q \subseteq B$. This distinction is not hypothetical; Section 4 presents an infinite dimensional example where $Q \cap S$ is empty.

Corollary 2.4. $Q$ is never empty. When $\mathcal{H}$ is finite dimensional, any $q \in Q$ satisfies $\|q\|=1$.

Proof. This follows from Fact 2.3 and the fact that $Q$ is defined as the weak accumulation points of a sequence in $S$.

The Kadec-Klee property, also called the Radon-Riesz property, holds for Hilbert spaces [5, Proposition 3.32].

Fact 2.5 (Kadec-Klee). Assume a sequence $\left\{q^{k}\right\}_{k \geq 0} \subset \mathcal{H}$ satisfies $\left\|q^{k}\right\|=1$ and $q^{k} \rightarrow q$. If $\|q\|=1$ then $q^{k} \rightarrow q$. If $\|q\|<1$ then $q^{k} \nrightarrow q$.

Weak but not strong cosmic limits arguably tell us less about the direction in which $x^{k}$ diverges to infinity, especially when the weak cosmic limit is 0 as in Section 4 . In finite dimensions, Corollary 2.4 states that $Q \subseteq S$. In infinite dimensions, Corollary 2.4 and Fact 2.5 states $Q \cap S$ are the strong cosmic accumulation points and $Q \backslash S$ are the weak cosmic accumulation points.

Again, this paper focuses on non-expansive operators $T$ with minimal displacement vectors $v=0$. We do so, because strong cosmic convergence immediately follows from known results when $v \neq 0$.

Fact 2.6. Assume $T: \mathcal{H} \rightarrow \mathcal{H}$ is non-expansive and has no fixed point. Write $v$ for the minimal displacement vector of $T$. If $v \neq 0$, then

$$
\left(1 /\left\|x^{k}\right\|\right) x^{k} \rightarrow-v /\|v\| .
$$

Proof. Theorem 2 of $\left[10\right.$ states that $(1 / k) x^{k} \rightarrow-v$, which also implies $(1 / k)\left\|x^{k}\right\| \rightarrow\|v\|$. The lemma follows from dividing the two limits.

Corollary 2.7. Assume $T: \mathcal{H} \rightarrow \mathcal{H}$ is non-expansive and has no fixed point. If $\left\{x^{k}\right\}_{k \geq 0}$ does not cosmically converge or cosmically converges weakly but not strongly, then $v=0$. 
Proof. If $v \neq 0$, then $\left\{x^{k}\right\}_{k \geq 0}$ cosmically converges strongly by Fact 2.6. The corollary is the contrapositive of this statement.

By Corollary 2.7 the operators presented in Section 3 and 4 have minimal displacement vectors $v=0$.

\section{Counter example: cosmic divergence}

In this section, we present an operator on $\mathbb{R}^{2}$ for which cosmic convergence fails. That this operator has minimal displacement vector $v=0$ follows from Corollary 2.7.

\subsection{General construction}

Consider the function $f: \mathbb{R}^{2} \rightarrow \mathbb{R}$ defined as

$$
f(x, y)=\max \{\Phi(x), \Psi(y)\}
$$

where

$$
\Phi(x)=\int_{0}^{x} \varphi\left(x^{\prime}\right) d x^{\prime}, \quad \Psi(y)=\int_{0}^{y} \psi\left(y^{\prime}\right) d y^{\prime} .
$$

Assume $\varphi$ and $\psi$ are nondecreasing functions from $\mathbb{R}$ to $[-1,0)$. This makes $\Phi$ and $\Psi$ (continuous) strictly decreasing convex functions from $\mathbb{R}$ to $\mathbb{R}$, and this makes $f$ convex. (Cf. Example 8.22 and Proposition 9.3 of [3] or Theorem 5.5 and Section 24 of [11.)

Consider the proximal operator

$$
\operatorname{Prox}_{f}\left(x^{\prime}, y^{\prime}\right)=\underset{x, y \in \mathbb{R}}{\arg \min }\left\{f(x, y)+\frac{1}{2}\left(x-x^{\prime}\right)^{2}+\frac{1}{2}\left(y-y^{\prime}\right)^{2}\right\}
$$

and the iteration

$$
\left(x_{k+1}, y_{k+1}\right)=\operatorname{Prox}_{f}\left(x_{k}, y_{k}\right),
$$

with starting points $x_{0}=y_{0}=0$.

Lemma 3.1 (9]). The operator

$$
\operatorname{Prox}_{f}: \mathbb{R}^{2} \rightarrow \mathbb{R}^{2}
$$

is well-defined (the argmin exists and is unique) and is non-expansive.

Define the curve

$$
\Gamma=\left\{(x, y) \in \mathbb{R}^{2} \mid \Phi(x)=\Phi(y) \leq 0\right\} .
$$

Note that $\Phi(0)=\Psi(0)=0$, and thus $\left(x_{0}, y_{0}\right) \in \Gamma$. In fact, $\left\{\left(x_{k}, y_{k}\right)\right\}_{k \geq 0} \subset \Gamma$.

Lemma 3.2. If $\Phi\left(x^{\prime}\right)=\Psi\left(y^{\prime}\right)$ then

$$
(x, y)=\operatorname{Prox}_{f}\left(x^{\prime}, y^{\prime}\right)
$$

satisfies

$$
\Phi(x)=\Psi(y)
$$


Proof. Without loss of generality, assume for contradiction that $\Phi(x)>\Psi(y)$. By definition $\operatorname{Prox}_{f}$, we have

$$
\Psi(y)<\Phi(x)=\max \{\Phi(x), \Psi(y)\} \leq \max \left\{\Phi\left(x^{\prime}\right), \Psi\left(y^{\prime}\right)\right\}=\Psi\left(y^{\prime}\right) .
$$

So $\Psi(y)<\Phi(x) \leq \Psi\left(y^{\prime}\right)$ and, since $\Psi$ is continuous, there is a $\theta \in[0,1)$ such that

$$
\Psi\left(\theta y+(1-\theta) y^{\prime}\right)=\Phi(x) .
$$

Since $\Psi(y)<\Psi\left(y^{\prime}\right)$, we have $y \neq y^{\prime}$. This means

$$
\Phi(x)=\max \{\Phi(x), \Psi(y)\}=\max \left\{\Phi(x), \Psi\left(\theta y+(1-\theta) y^{\prime}\right)\right\}
$$

and therefore

$$
\begin{aligned}
& f(x, y)+\frac{1}{2}\left(x-x^{\prime}\right)^{2}+\frac{1}{2}\left(y-y^{\prime}\right)^{2} \\
& >f\left(x, \theta y+(1-\theta) y^{\prime}\right)+\frac{1}{2}\left(x-x^{\prime}\right)^{2}+\frac{1}{2}\left(\left(\theta y+(1-\theta) y^{\prime}\right)-y^{\prime}\right)^{2} \\
& =f\left(x, \theta y+(1-\theta) y^{\prime}\right)+\frac{1}{2}\left(x-x^{\prime}\right)^{2}+\frac{1}{2} \theta^{2}\left(y-y^{\prime}\right)^{2}
\end{aligned}
$$

This contradicts the fact that $(x, y)=\operatorname{Prox}_{f}\left(x^{\prime}, y^{\prime}\right)$ is defined as the minimizer.

Fact 3.3 (Theorem 2.1 of [7]). The iteration (3.1) satisfies

$$
f\left(x_{k}, y_{k}\right) \rightarrow \inf _{x, y \in \mathbb{R}} f(x, y)
$$

as $k \rightarrow \infty$. (To be clear, this holds even when $\inf _{x, y \in \mathbb{R}} f(x, y)$ has no minimizer and when $\inf _{x, y \in \mathbb{R}} f(x, y)=-\infty$.)

By Lemma 3.2 and Fact 3.3 ,

$$
f\left(x_{k}, y_{k}\right)=\Phi\left(x_{k}\right)=\Psi\left(y_{k}\right) \rightarrow \inf _{x, y \in \mathbb{R}} f(x, y)=\max \left\{\inf _{x \in \mathbb{R}} \Phi(x), \inf _{y \in \mathbb{R}} \Psi(y)\right\}
$$

as $k \rightarrow \infty$. Because $\Phi$ and $\Psi$ are strictly decreasing functions, the minimum of $f(x, y)$ is not attained. Therefore, $\left\{\left(x_{k}, y_{k}\right)\right\}_{k \geq 0}$ has no cluster points, and $x_{k} \rightarrow \infty$ and/or $y_{k} \rightarrow \infty$.

We can think of $\Gamma$ as a 1-dimensional curve in $\mathbb{R}^{2}$ starting at $(0,0)$ and going off to infinity. The sequence $\left\{\left(x_{k}, y_{k}\right)\right\}_{k \geq 0}$ stays on and moves along the curve $\Gamma$ without clustering anywhere. Lemma 3.5 implies that the sequence $\left\{\left(x_{k}, y_{k}\right)\right\}_{k \geq 0}$ traverses $\Gamma$ in small increments of size at most 1 . Conversely, Lemma 3.6 states that for any point $\Gamma$, there is a point among $\left\{\left(x_{k}, y_{k}\right)\right\}_{k \geq 0}$ with distance at most 1 .

Lemma 3.4 (Corollary 17.19 of [3]). Assume a (closed) convex function $f$ : $\mathbb{R}^{n} \rightarrow \mathbb{R}$ is L-Lipschitz continuous. Then any $g \in \partial f(x)$ for any $x \in \mathbb{R}^{n}$ satisfies $\|g\| \leq L$.

Lemma 3.5. For all $x, y \in \mathbb{R}$

$$
\left\|(x, y)-\operatorname{Prox}_{f}(x, y)\right\| \leq 1 .
$$


Proof. Since $\Phi(x)$ and $\Psi(y)$ are defined to be antiderivatives of $\varphi(x)$ and $\psi(y)$, functions with absolute magnitude at most 1, they are 1-Lipschitz continuous functions. This makes $f(x, y)=\max \{\Phi(x), \Psi(y)\}$ 1-Lipschitz continuous as well. By Lemma 3.4. any $g \in \partial f(x, y)$ for any $x, y \in \mathbb{R}$ satisfies $\|g\| \leq 1$.

Write

$$
\left(x_{+}, y_{+}\right)=\operatorname{Prox}_{f}(x, y) .
$$

Then the optimality conditions of the minimization defining $\operatorname{Prox}_{f}$ gives us

$$
g=\left(x-x_{+}, y-y_{+}\right) \in \partial f\left(x_{+}, y_{+}\right) .
$$

Since $\|g\| \leq 1$, we have $\left\|\left(x-x_{+}, y-y_{+}\right)\right\| \leq 1$.

Lemma 3.6. For any $(\xi, \zeta) \in \Gamma$, there is a $k \geq 0$ such that

$$
\left\|(\xi, \zeta)-\left(x_{k}, y_{k}\right)\right\| \leq 1
$$

Proof. Fact 3.3 tells us

$$
f\left(x_{k}, y_{k}\right) \rightarrow \max \left\{\inf _{x \in \mathbb{R}} \Phi(x), \inf _{y \in \mathbb{R}} \Psi(y)\right\}
$$

as $k \rightarrow \infty$. Since $\Phi$ and $\Psi$ are strictly decreasing, $f\left(x_{k}, y_{k}\right)$ never attains the limit for finite $k$. Since $(\xi, \zeta) \in \Gamma$, we have $\Phi(\xi)=\Psi(\zeta) \leq 0$. Finally, since $f\left(x_{0}, y_{0}\right)=0$, there is a $k$ such that

$$
f\left(x_{k}, y_{k}\right) \geq \Phi(\xi)=\Psi(\zeta)>f\left(x_{k+1}, y_{k+1}\right) .
$$

By Lemma 3.2, this further implies

$$
\begin{aligned}
& \Phi\left(x_{k}\right) \geq \Phi(\xi)>\Phi\left(x_{k+1}\right) \\
& \Psi\left(y_{k}\right) \geq \Psi(\zeta)>\Psi\left(y_{k+1}\right) .
\end{aligned}
$$

Since $\Phi$ and $\Psi$ are strictly decreasing univariate functions, we have $x_{k} \leq \xi<$ $x_{k+1}$ and $y_{k} \leq \zeta<y_{k+1}$. With Lemma 3.5 we have

$$
\left\|\left(x_{k}, y_{k}\right)-(\xi, \zeta)\right\|^{2} \leq\left\|\left(x_{k}, y_{k}\right)-\left(x_{k+1}, y_{k+1}\right)\right\|^{2} \leq 1 \text {. }
$$

\subsection{Design of $\varphi$ and $\psi$}

So the sequence $\left\{\left(x_{k}, y_{k}\right)\right\}_{k \geq 0}$ stays on and moves along the curve $\Gamma$. We now design $\varphi$ and $\psi$ so that there a sequence $\left\{\left(\xi_{n}, \zeta_{n}\right)\right\}_{n \geq 0} \subset \Gamma$ with more than one cosmic accumulation point. Roughly speaking, this means $\Gamma$ goes off to infinity with no single cosmic direction, and this will imply $\left\{\left(x_{k}, y_{k}\right)\right\}_{k \geq 0}$ has no single cosmic limit. This argument is made formal in Section 3.3 .

Let

$$
\xi_{n}=\sum_{i=1}^{n} i^{i}
$$

for $n=1,2, \ldots$ and $\xi_{0}=0$. Also let

$$
\zeta_{n}=\sum_{i=1}^{n} \frac{2}{3+(-1)^{i}} i^{i}
$$


for $n=1,2, \ldots$ and $\zeta_{0}=0$. For $n>1$, we have

$$
\xi_{n}=\sum_{i=1}^{n} i^{i} \leq \sum_{i=1}^{n} n^{i} \leq \sum_{i=0}^{n} n^{i}=\frac{n^{n+1}-1}{n-1} \leq \frac{n^{n+1}}{n-1}
$$

and

$$
\zeta_{n} \leq \xi_{n} \leq \frac{n^{n+1}}{n-1} .
$$

For $n>1$, we have

$$
0 \leq \frac{\xi_{n}}{\xi_{n+1}-\xi_{n}}=\frac{\xi_{n}}{(n+1)^{n+1}} \leq \frac{n^{n+1}}{(n-1)(n+1)^{n+1}} \leq \frac{1}{n-1},
$$

where the first inequality and equality follows from the definition of $\left\{\xi_{n}\right\}_{n \geq 0}$, the second inequality follows from the bound on $\xi_{n}$, and the third inequality follows from $n^{n+1} \leq(n+1)^{n+1}$. Likewise, we have

$$
0 \leq \frac{\zeta_{n}}{\zeta_{n+1}-\zeta_{n}} \leq \frac{\zeta_{n}}{(n+1)^{n+1} / 2} \leq \frac{2 n^{n+1}}{(n-1)(n+1)^{n+1}} \leq \frac{2}{n-1}
$$

for $n>1$. These inequalities say that the increment from $\xi_{n}$ to $\xi_{n+1}$ is much larger than $\xi_{n}$ (and the same is true for $\zeta_{n}$ ).

Define

$$
\varphi(x)= \begin{cases}-1 & \text { for } x<0 \\ -\frac{1}{n^{n}} & \text { for } x \in\left[\xi_{n-1}, \xi_{n}\right), \quad \text { for } n=1,2, \ldots\end{cases}
$$

and

$$
\psi(y)= \begin{cases}-1 & \text { for } y<0 \\ -\frac{3+(-1)^{n}}{2} \frac{1}{n^{n}} & \text { for } y \in\left[\zeta_{n-1}, \zeta_{n}\right), \quad \text { for } n=1,2, \ldots .\end{cases}
$$

Note that

$$
\begin{aligned}
\Phi\left(\xi_{n}\right) & =\sum_{i=1}^{n} \int_{\xi_{i-1}}^{\xi_{i}} \varphi\left(x^{\prime}\right) d x^{\prime} \\
& =\sum_{i=1}^{n}\left(-\frac{1}{i^{i}}\left(\xi_{i}-\xi_{i-1}\right)\right) \\
& =\sum_{i=1}^{n}(-1)=-n,
\end{aligned}
$$

and, by the same reasoning, $\Psi\left(\zeta_{n}\right)=-n$. Therefore, $\Phi\left(\xi_{n}\right)=\Psi\left(\zeta_{n}\right)=-n$ and $\left(\xi_{n}, \zeta_{n}\right) \in \Gamma$ for $n=1,2, \ldots$

The sequence $\left\{\left(\xi_{n}, \zeta_{n}\right)\right\}_{n \geq 0}$ diverges to infinity, i.e., $\left\|\left(\xi_{n}, \zeta_{n}\right)\right\| \rightarrow \infty$, and has two cosmic accumulation points. To see why, note that

$$
\begin{aligned}
\frac{\xi_{n+1}}{\zeta_{n+1}} & =\frac{\xi_{n+1}-\xi_{n}+\xi_{n}}{\zeta_{n+1}-\zeta_{n}+\zeta_{n}} \\
& =\frac{\xi_{n+1}-\xi_{n}}{\zeta_{n+1}-\zeta_{n}} \frac{1+\xi_{n} /\left(\xi_{n+1}-\xi_{n}\right)}{1+\zeta_{n} /\left(\zeta_{n+1}-\zeta_{n}\right)} \\
& =\frac{3+(-1)^{n+1}}{2} \frac{1+\mathcal{O}(1 / n)}{1+\mathcal{O}(1 / n)} .
\end{aligned}
$$


So

$$
\frac{\xi_{2 m}}{\zeta_{2 m}} \rightarrow 2 \quad \frac{\xi_{2 m+1}}{\zeta_{2 m+1}} \rightarrow 1
$$

as $m \rightarrow \infty$. This tells us $\left\{\left(\xi_{n}, \zeta_{n}\right)\right\}_{n=0,2,4, \ldots}$ has the cosmic limit $(2 / \sqrt{5}, 1 / \sqrt{5})$ and $\left\{\left(\xi_{n}, \zeta_{n}\right)\right\}_{n=1,3,5, \ldots}$ has the cosmic limit $(1 / \sqrt{2}, 1 / \sqrt{2})$. We conclude that the whole sequence $\left\{\left(\xi_{n}, \zeta_{n}\right)\right\}_{n \geq 0}$ has two cosmic accumulation points.

\subsection{Cosmic divergence}

Since $\left\{\left(\xi_{n}, \zeta_{n}\right)\right\}_{n \geq 0} \subset \Gamma$, Lemma 3.6 says there is a subsequence

$$
\left\{\left(x_{k_{n}}, y_{k_{n}}\right)\right\}_{n \geq 0} \subset\left\{\left(x_{k}, y_{k}\right)\right\}_{k \geq 0}
$$

such that

$$
\left\|\left(x_{k_{n}}, y_{k_{n}}\right)-\left(\xi_{n}, \zeta_{n}\right)\right\| \leq 1
$$

for $n=0,1, \ldots$ By Lemma 2.1. $\left\{\left(x_{k_{n}}, y_{k_{n}}\right)\right\}_{n \geq 0}$ has the same cosmic accumulation points as $\left(\xi_{n}, \zeta_{n}\right)$. Thus we conclude that the whole sequence $\left\{\left(x_{k}, y_{k}\right)\right\}_{k \geq 0}$ has more than 1 cosmic accumulation point.

\section{Counter example: weak, but not strong, cosmic convergence}

In this section, we present an operator on an infinite dimensional Hilbert space for which its fixed-point iteration cosmically converges to 0 weakly, i.e., the iterates of 1.1 satisfy

$$
\left(1 /\left\|x^{k}\right\|\right) x^{k} \rightarrow 0 .
$$

This example shows that weak cosmic convergence is indeed possible in infinite dimensions and that a weak cosmic limit may not be very informative in understanding the direction in which $\left\{x^{k}\right\}_{k \geq 0}$ diverges to infinity. That this operator has minimal displacement vector $v=0$ follows from Corollary 2.7.

\subsection{Univariate building block}

We first build and analyze a non-expansive operator on $\mathbb{R}$ and later use it to build the full example.

Consider the convex function $\varphi: \mathbb{R} \rightarrow \mathbb{R}$ defined as

$$
\varphi(x)= \begin{cases}1-x & \text { for } x<0 \\ e^{-x} & \text { for } x \geq 0\end{cases}
$$

Let $\alpha \in(0,1]$. Then the gradient descent operator

$$
t(x)=x-\alpha \varphi^{\prime}(x)
$$

is non-expansive.

Consider the fixed-point iteration

$$
\begin{aligned}
x_{k+1} & =t\left(x_{k}\right) \\
& =x_{k}+\alpha e^{-x_{k}}
\end{aligned}
$$

with starting point $x_{0}=0$. It is easy to see that $x_{k} \geq 0$ for all $k=0,1, \ldots$ The iterates satisfy

$$
x_{k}=\log k+\mathcal{O}(1)
$$


Lemma 4.1. For $k=0,1, \ldots$, the $x_{k}$ iterates of 4.2 satisfy

$$
\log (k+1)+\log \alpha \leq x_{k} \leq \log (k+1)+\log 2 .
$$

Proof. We prove this by induction. Since $\alpha \in(0,1]$ and $x_{0}=0$, the base case holds trivially.

Now assume the statement holds for $k$. Then

$$
\begin{aligned}
x_{k} & \geq \log \alpha+\log (k+1) \\
x_{k+1} & \geq \log \alpha+\log (k+1)+\frac{1}{k+1} \\
& \geq \log \alpha+\log (k+2) .
\end{aligned}
$$

The second line follows from plugging the first line into the function $x+\alpha e^{-x}$, which is nondecreasing on $x \in[\log \alpha, \infty)$. The third line follows from the inequality

$$
\log (k+1)+\frac{1}{k+1} \geq \log (k+2),
$$

which in turn follows from upper bounding the concave function log with its first order Taylor expansion about $k+1$.

Similarly, we have

$$
\begin{aligned}
x_{k} & \leq \log (k+1)+\log 2 \\
x_{k+1} & \leq \log (k+1)+\log 2+\frac{\alpha}{2(k+1)} \\
& \leq \log (k+1)+\log 2+\frac{1}{2(k+1)} \\
& \leq \log (k+1)+\log 2+\frac{1}{k+2} \\
& \leq \log (k+2)+\log 2 .
\end{aligned}
$$

Again, the second line follows from plugging the first line into $x+\alpha e^{-x}$. The third line simply follows from $\alpha \leq 1$. The fourth line follows from the inequality

$$
\frac{1}{2(k+1)} \leq \frac{1}{k+2}
$$

for $k \geq 0$. The fifth line follows from

$$
\log (k+2)-\frac{1}{k+2} \geq \log (k+1),
$$

which in turn follows from upper bounding the concave function log with its first order Taylor expansion about $k+2$.

\subsection{Main construction}

Consider the standard $\ell^{2}$ space, the space of square-summable (real) sequences with inner product

$$
\langle x, y\rangle=\sum_{i=1}^{\infty} x_{i} y_{i} .
$$


Consider the convex function $f: \ell^{2} \rightarrow \mathbb{R}$

$$
f(x)=\sum_{i=1}^{\infty} \frac{1}{i^{2}} \varphi\left(x_{i}\right),
$$

where $\varphi$ is as defined in 4.1). Consider the operator

$$
T(x)=x-\nabla f(x)
$$

which is

$$
(T(x))_{i}=x_{i}-\frac{1}{i^{2}} \varphi^{\prime}\left(x_{i}\right)
$$

for $i=1,2, \ldots$ Since the mapping of each coordinate $x_{i} \mapsto x_{i}-\left(1 / i^{2}\right) \varphi^{\prime}\left(x_{i}\right)$ is non-expansive, $T: \ell^{2} \rightarrow \ell^{2}$ is non-expansive.

Now consider the fixed-point iteration

$$
x^{k+1}=T\left(x^{k}\right)
$$

with starting point $x^{0}=0 \in \ell^{2}$. This simplifies to

$$
\left(x^{k+1}\right)_{i}=x_{i}^{k}+\left(1 / i^{2}\right) e^{-x_{i}^{k}}
$$

for $i=1,2, \ldots$ Lemma 4.1 states

$$
x_{i}^{k}=\log k+\mathcal{O}(1)
$$

as $k \rightarrow \infty$ for fixed $i$.

This coordinate-wise rate implies

$$
\left(1 /\left\|x^{k}\right\|\right) x_{i}^{k} \rightarrow 0
$$

as $k \rightarrow \infty$ for fixed $i$. To see why, pick any $N \in \mathbb{N}$ and we get

$$
\left\|x^{k}\right\|^{2} \geq\left(\left|x_{1}^{k}\right|^{2}+\cdots+\left|x_{N}^{k}\right|^{2}\right) \geq N(\log k)^{2}+\mathcal{O}(1) .
$$

Therefore

$$
\limsup _{k \rightarrow \infty}\left(1 /\left\|x^{k}\right\|\right)\left|x_{i}^{k}\right| \leq 1 / \sqrt{N}
$$

and we let $N \rightarrow \infty$ to conclude $\left(1 /\left\|x^{k}\right\|\right) x_{i}^{k} \rightarrow 0$. Since each coordinate of the sequence $\left\{\left(1 /\left\|x^{k}\right\|\right) x^{k}\right\}_{k \geq 0} \subset \ell^{2}$ converges to 0 , we have

$$
\left(1 /\left\|x^{k}\right\|\right) x^{k} \rightarrow 0 .
$$

On the other hand, Fact 2.5 and the fact that $\left(1 /\left\|x^{k}\right\|\right) x^{k}$ has unit norm for $k>0$ implies

$$
\left(1 /\left\|x^{k}\right\|\right) x^{k} \nrightarrow \rightarrow 0 .
$$

Therefore, $\left\{x^{k}\right\}_{k \geq 0}$ cosmically converges to 0 weakly but not strongly.

\section{Positive results}

In this section, we presents positive results towards characterizing cosmic accumulation points. Section 5.1 states the results and Section 5.2 provides their interpretation. Section 5.3 relates these results to past work. 


\subsection{Results}

Theorem 5.1. For any $q \in Q$ and $x \in \mathcal{H}$,

$$
\langle T(x)-x, q\rangle \geq 0 \text {. }
$$

So $q$ defines a separating hyperplane between $\overline{\operatorname{ran}(I-T)}$ and $\{0\}$, if $q \neq 0$.

Proof. By definition of $Q$, the iterates $\left\{x^{k}\right\}_{k \geq 0}$ of 1.1 has a subsequence $\left\{x^{k_{j}}\right\}_{j \geq 0}$ such that

$$
\left(1 /\left\|x^{k_{j}}\right\|\right) x^{k_{j}} \rightarrow q, \quad\left\|x^{k_{j}}\right\| \rightarrow \infty, \quad\left\|T\left(x^{k_{j}}\right)-x^{k_{j}}\right\| \rightarrow 0 .
$$

For any $x \in \mathcal{H}$, we have

$$
\begin{aligned}
\left\|x-x^{k_{j}}\right\|^{2} \geq \| & \left\|(x)-T\left(x^{k_{j}}\right)\right\|^{2} \\
= & \left\|x-x^{k_{j}}\right\|^{2}+2\left\langle x-x^{k_{j}}, T(x)-x-T\left(x^{k_{j}}\right)+x^{k_{j}}\right\rangle \\
& \quad+\left\|T(x)-x-T\left(x^{k_{j}}\right)+x^{k_{j}}\right\|^{2} .
\end{aligned}
$$

We reorganize this to get

$$
\begin{aligned}
-\frac{1}{\left\|x^{k_{j}}\right\|} \| T(x) & -x-T\left(x^{k_{j}}\right)+x^{k_{j}} \|^{2} \\
& \geq \frac{2}{\left\|x^{k_{j}}\right\|}\left\langle x-x^{k_{j}}, T(x)-x-T\left(x^{k_{j}}\right)+x^{k_{j}}\right\rangle .
\end{aligned}
$$

By taking the limit $j \rightarrow \infty$ we get

$$
0 \geq-\langle q, T(x)-x\rangle \text {. }
$$

Corollary 5.2. If $q \in Q$ and $q \neq 0$, then the iterates of (1.1 satisfy

$$
\left\langle x^{k}, q\right\rangle \rightarrow \infty
$$

monotonically as $k \rightarrow \infty$ for any starting point $x^{0} \in \mathcal{H}$.

Proof. Since

$$
\left\langle x^{k+1}-x^{k}, q\right\rangle \geq 0
$$

by Theorem 5.1. the scalar sequence $\left\{\left\langle x^{k}, q\right\rangle\right\}_{k \geq 0}$ is nondecreasing. Since $q \in Q$, there is a subsequence $\left\{x^{k_{j}}\right\}_{j \geq 0}$ such that

$$
\left(1 /\left\|x^{k_{j}}\right\|\right) x^{k_{j}} \rightarrow q .
$$

Therefore, we have

$$
\left(1 /\left\|x^{k_{j}}\right\|\right)\left\langle x^{k_{j}}, q\right\rangle \rightarrow\langle q, q\rangle
$$

and $\left\langle x^{k_{j}}, q\right\rangle \rightarrow \infty$ as $j \rightarrow \infty$. Since $\left\{\left\langle x^{k}, q\right\rangle\right\}_{k \geq 0}$ is nondecreasing, and since a subsequence of it converges to $\infty$, the whole sequence converges to $\infty$.

Corollary 5.3. If $q_{1}, q_{2} \in Q$ then

$$
\left\langle q_{1}, q_{2}\right\rangle \geq 0 .
$$


Proof. Assume $q_{1} \neq 0$ as otherwise the result is trivial. Since $q_{2} \in Q$, there is a subsequence $\left\{x^{k_{j}}\right\}_{j \geq 0}$ such that

$$
\left(1 /\left\|x^{k_{j}}\right\|\right) x^{k_{j}} \rightarrow q_{2} .
$$

By Corollary 5.2 we have

$$
\left\langle q_{1}, x^{k_{j}}\right\rangle \rightarrow \infty
$$

and therefore

$$
\lim _{j \rightarrow \infty} \frac{1}{\left\|x_{k_{j}}\right\|}\left\langle q_{1}, x^{k_{j}}\right\rangle=\left\langle q_{1}, q_{2}\right\rangle \geq 0 .
$$

Given a set $A \subseteq \mathcal{H}$, write $A^{*}$ for the dual cone of $A$, i.e.,

$$
A^{*}=\{v \in \mathcal{H} \mid\langle v, a\rangle \geq 0 \text { for any } a \in A\} .
$$

Theorem 5.4. If $T: \mathcal{H} \rightarrow \mathcal{H}$ is non-expansive, then

$$
Q \subseteq \overline{\operatorname{cone}(\operatorname{ran}(T-I))} \cap(\overline{\operatorname{cone}(\operatorname{ran}(T-I))})^{*} \subseteq Q^{*} .
$$

Proof. That $Q \subseteq \overline{\operatorname{cone}(\operatorname{ran}(T-I))}$ follows from Theorem 2.6 of 4 . That $Q \subseteq(\overline{\operatorname{cone}(\operatorname{ran}(T-I))}) *$ follows from Theorem 5.1. The final inclusion follows from the fact that when $B$ is cone,

$$
A \subseteq B \cap B^{*}
$$

implies $B^{*} \subseteq A^{*}$ and $B \subseteq A^{*}$, cf. Proposition 6.24 of [3].

\subsection{Interpretation via points at infinity}

In addition to the interpretation as a separating hyperplane, a $q \in Q$ such that $q \neq 0$ has an interpretation as a fixed point at infinity.

In the classical case where $S: \mathcal{H} \rightarrow \mathcal{H}$ is non-expansive and has fixed point $x^{\star} \in \mathcal{H}$, we have

$$
\left\|S(x)-x^{\star}\right\| \leq\left\|x-x^{\star}\right\|
$$

for any $x \in \mathcal{H}$. In other words, $S(x)$ stays within the closed ball of radius $\left\|x-x^{\star}\right\|$ centered about $x^{\star}$.

We have an analogous geometric interpretation of Theorem 5.1. $T(x)$ stays within the half-space

$$
\{y \in \mathcal{H} \mid\langle q, y\rangle \geq\langle q, x\rangle\},
$$

for any $x \in \mathcal{H}$. See Figure 1 . This is like saying $T(x)$ stays within the closed ball of radius $\infty$ centered about $\infty \cdot q$, and this is the intuition of the proof of Theorem 5.1. We can say all steps of the fixed-point iteration (1.1) proceeds forward infinitely in the direction $q$ by Corollary 5.2 .

Write $B \subset \mathcal{H}$ for the closed unit ball, int $B \subset \mathcal{H}$ for the open unit ball, and $S \subset \mathcal{H}$ for the unit sphere. Consider the one-to-one map $f: \mathcal{H} \rightarrow \operatorname{int} B$ defined as

$$
f(x)=\frac{1}{1+\|x\|} x .
$$

It is interesting to map the geometric interpretation in $\mathcal{H}$ onto $\operatorname{int} B$ via $f$. 


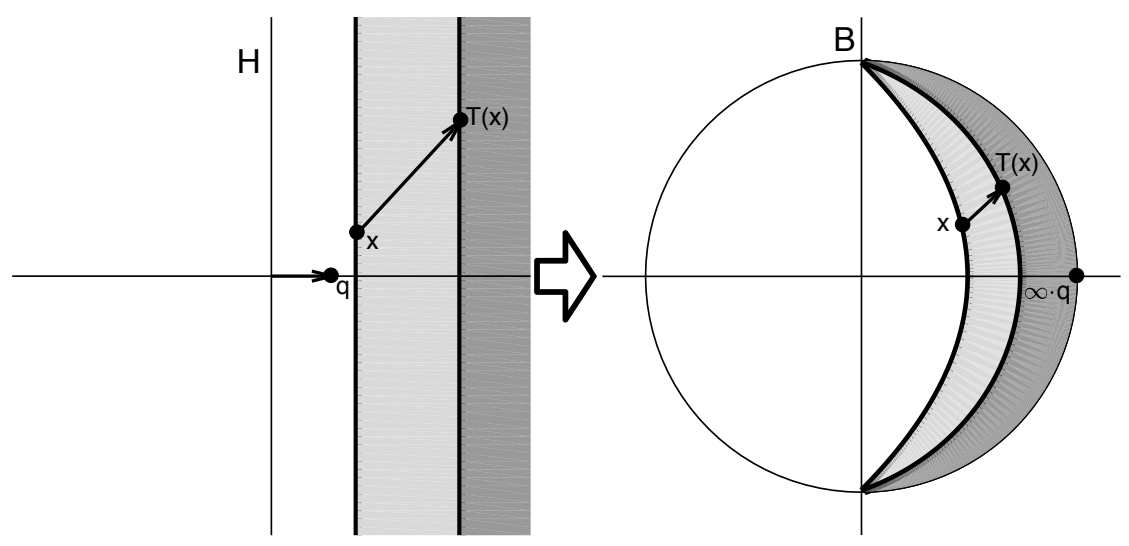

FiguRE 1. Geometric interpretation Theorem 5.1 on $\mathcal{H}$ and on $B$. The right is the image of the left under $f$ as defined in (5.1).

In Figure 1, the half-space is mapped into a crescent-shaped image under $f$, and $\infty \cdot q$ is mapped to $\lim _{a \rightarrow \infty} f(a q) \in S$, the outer center of the crescent shape. In the fixed-point iteration (1.1), the half-space defined by $x^{k}$ moves forward in the direction $q$ in $\mathcal{H}$. This corresponds to the crescent shape becoming thinner, but not smaller, in $B$.

\subsection{Relationship to existing work}

Lins, Gaubert, and Vigeral presented results related to Theorem 5.1. Under the setup and notation of this paper, they are as follows.

Theorem 5.5 (Theorem 1.2 of [8]). Let $T: \mathbb{R}^{n} \rightarrow \mathbb{R}^{n}$ be non-expansive with no fixed points. Then there is a $\nu \in \mathbb{R}^{n}$ such that

$$
\left\langle\nu, x^{k}\right\rangle \rightarrow \infty
$$

for any starting point $x^{0}$.

Theorem 5.6 (Corollary 21 of [6]). Let $T: \mathbb{R}^{n} \rightarrow \mathbb{R}^{n}$ be non-expansive with no fixed points. Then there is a $\nu \in \mathbb{R}^{n}$ such that

$$
\left\langle\nu, x^{0}\right\rangle \leq\left\langle\nu, x^{1}\right\rangle \leq\left\langle\nu, x^{2}\right\rangle \leq \ldots
$$

for any starting point $x^{0}$.

In their original papers, Theorem 5.5 and 5.6 are more general, as they hold for finite dimensional Banach spaces. In the case of finite dimensional Hilbert spaces, Corollaries 5.2 and 2.4 subsumes Theorem 5.5 and 5.6 Furthermore, Corollary 5.2 is more constructive than Theorem 5.5 and 5.6, as it provides an explicit characterization of the linear functional $\nu$.

Bauschke, Douglas, and Moursi presented various results towards understanding the conditions for cosmic convergence, including Theorem 5.7 . The presented Theorem 5.4 builds on and strengthens Theorem 5.7 . 
Theorem 5.7 (Theorem 2.6 of [4]). If $T: \mathcal{H} \rightarrow \mathcal{H}$ is non-expansive, then

$$
Q \subseteq \overline{\operatorname{cone}(\operatorname{ran}(T-I))} \text {. }
$$

\section{Conclusion}

We discussed counter examples showing that the cosmic convergence conjecture is false and that weak cosmic convergence can happen in infinite dimensions. We then discussed some new positive results relating to cosmic convergence, which allow us to interpret cosmic accumulation points as fixed points at infinity.

Despite the counter examples, however, the author still believes the notion of cosmic convergence is useful in understanding the asymptotic behavior of the fixed-point iteration (1.1). In fact, the author still conjectures that cosmic convergence usually holds. Finding general conditions that ensure cosmic convergence is an interesting future direction.

One potentially interesting and fruitful approach may be to focus on firmly non-expansive operators. Many operators that arise from convex optimization are not just non-expansive but are furthermore firmly non-expansive. At the same time, however, firm non-expansivity will not make the question of cosmic convergence trivial. After all, the counter examples of Section 3 and 4 are firmly non-expansive operators. Also it does not seem like we can strengthen Theorem 5.1 by assuming firm non-expansivity.

\section{References}

[1] C. Aliprantis and K. Border, Infinite Dimensional Analysis: A Hitchhiker's Guide. 3rd Edition, Springer, 2006.

[2] J.-B. Baillon, R. E. Bruck, and S. Reich, On the Asymptotic Behavior of Nonexpansive Mappings and Semigroups in Banach Spaces. Houston Journal of Mathematics 4:1 (1978), 1-9.

[3] H. H. Bauschke and P. L. Combettes, Convex Analysis and Monotone Operator Theory in Hilbert Spaces. Second Edition, Springer, 2017.

[4] H. H. Bauschke, G. R. Douglas, and W. M. Moursi, On a result of Pazy concerning the asymptotic behaviour of non-expansive mappings. Journal of Fixed Point Theory and Applications 18:2 (2016), 297-307.

[5] H. Brezis, Functional Analysis, Sobolev Spaces and Partial Differential Equations, Springer New York, 2010.

[6] S. Gaubert and G. Vigeral, A maximin characterisation of the escape rate of non-expansive mappings in metrically convex spaces. Mathematical Proceedings of the Cambridge Philosophical Society 152:2 (2012) 341-363.

[7] O. Güler, On the Convergence of the Proximal Point Algorithm for Convex Minimization. SIAM Journal on Control and Optimization 29:2 (1991), 403419. 
[8] B. Lins, Asymptotic Behavior of non-expansive mappings in finite dimensional normed spaces. Proceedings of the American Mathematical Society 137:7 (2009), $2387-2392$.

[9] J. J. Moreau, Proximité et dualité dans un espace Hilbertien. Bulletin de la Société Mathématique de France 93 (1965): 273-299.

[10] A. Pazy, Asymptotic behavior of contractions in Hilbert space. Israel Journal of Mathematics 9:2 (1971), 235-240.

[11] R. T. Rockafellar, Convex Analysis, Princeton University Press, 1970.

Ernest K. Ryu

7324 Mathematical Sciences,

Los Angeles, CA 90095

United States

e-mail: eryu@math.ucla.edu 